\title{
Comparative Evaluation of Different Phenotypic Methods for Biofilm Formation among Uropathogenic $E$. coli (UPEC)
}

\author{
Rashmi P Mahale ${ }^{1}$, M. N. Sumana ${ }^{2 *}$, K. Anuradha ${ }^{3}$ and Adline Princy ${ }^{4}$ \\ ${ }^{1}$ Dept. of Microbiology, JSS Medical College \& Hospital, JSSAHER, Mysore, India \\ ${ }^{2}$ Department of Microbiology, MMC, Mysore, India \\ ${ }^{3}$ Department of Biotechnology, Sastra Deemed University, Thanjavur, Tamil Nadu \\ *Corresponding author
}

\section{A B S T R A C T}

\section{Keywords}

Biofilm, Infections,

Decreased

susceptibility,

Phenotypic methods

Article Info

Accepted:

11 June 2020

Available Online:

10 July 2020
Biofilms have the ability to attach to both biotic and abiotic surfaces. With the advent in medical industry, use of indwelling prosthetic devices have increased abiotic surfaces providing opportunities for attachment by the biofilm forming bacteria and thus leading to infections. Treatment of infection by biofilm forming bacteria is difficult due to their decreased susceptibility to antibiotics. Hence, this study was aimed to compare the sensitivity and specificity of the three phenotypic methods for the detection of biofilms.

\section{Introduction}

Bacteria exist in two growth modes in nature, one as the planktonic form and the other as sessile aggregates called biofilm. Existence in aggregates as Biofilm confers the bacterial community certain survival advantage like evading the host immune responses, resistance to antibiotics, biofilm also entraps the nutrients for the utilizations by the microbial community ${ }^{1,2}$. Biofilm is defined as a structured community of bacterial cells enclosed in a self-produced polymeric matrix which is made up of extracellular polymeric substances (EPS). ${ }^{3}$ According to National Institute of health (NIH) $65 \%$ of microbial infections and $80 \%$ of the chronic infections are due to biofilm forming microorganisms. Biofilms have the ability to attach to both biotic and abiotic surfaces. With the advent in medical industry, use of indwelling prosthetic devices have increased abiotic surfaces providing opportunities for attachment by the biofilm forming bacteria and thus leading to infections associated with them like catheter associated blood stream infections and catheter associated urinary tract infections, ventilator associated pneumonia etc. 
Treatment of infection by biofilm forming bacteria is difficult due to their decreased susceptibility to antibiotics and unresponsiveness to the standard antimicrobial susceptibility test results which are directed against the planktonic forms, eradication of biofilm associated organisms requires the determination of biofilm eradicating concentrations which are routinely not done. Thus, it is important to screen the clinical isolates for biofilm forming capacity especially in chronic infections. There are various methods for the detection of biofilm forming capacity of microorganisms which includes various phenotypic methods like Congo-red agar method (CRA), tube method (TM), tissue culture plate method (TCP), electron microscopy, confocal scanning microscopy and bioluminescent assay and molecular methods like detection of biofilm associated genes. This study was under taken to compare the sensitivity and specificity of the three phenotypic methods for the detection of biofilms.

\section{Materials and Methods}

180 E. coli isolated from clinically suspected cases of Urinary tract infections were evaluated for biofilm forming capacity by three different phenotypic methods namely tissue culture plate method, tube adherence test and Congo red agar method. Biofilm production by each of the methods was graded as high, moderate, and weak, only high and moderate graded isolates were considered as biofilm producers, weak and no biofilm production by each method was considered non biofilm producers. Tissue culture method was considered as the gold standard for biofilm formation.

\section{Tissue culture plate method ${ }^{4}$}

Fresh isolates from agar plates were inoculated into brain heart infusion (BHI) broth with $2 \%$ sucrose and incubated for 24hrs at $37^{0} \mathrm{c}$. Broth was diluted 1:100with fresh medium, $0.2 \mathrm{ml}$ of this broth was inoculated into sterile flat-bottomed microtiter plates or tissue culture plates (TCP) only medium was inoculated in a well which served as control to check sterility of the medium and nonspecific binding of media. The TCPs were incubated for $24 \mathrm{hrs}$ at $37^{\circ} \mathrm{c}$. After incubation contents of each well was gently removed by taping the plates and then were washed 4 times with $0.2 \mathrm{ml}$ of phosphate buffer saline (PBS pH 7.2) to remove freefloating "planktonic" bacteria. Biofilms formed in wells were fixed with sodium acetate $(2 \%)$ for half an hour and stained with crystal violet $(0.1 \% \mathrm{w} / \mathrm{v})$ for half an hour. Excess stain was removed by thorough washing with deionized water and plates were kept for drying. Adherent E. coli formed biofilm on all side of the wells and were uniformly stained with crystal violet. Optical Density (OD) of stained wells were determined with a micro Enzyme-Linked Immunosorbent Assay auto reader at wavelength of $570 \mathrm{~nm}$ and were graded as per Christensen et al. The OD values were considered as an index of bacteria adhering to surface and forming biofilms.

Experiment was performed in triplicate; the data was then averaged and standard deviation was calculated. To compensate for background absorbance, the mean OD value obtained from media control well was deducted from all test values.

\section{Tube adherence method ${ }^{5}$}

Fresh isolates from agar plates were inoculated into $10 \mathrm{ml}$ brain heart infusion (BHI) broth with $2 \%$ sucrose and incubated for $24 \mathrm{hrs}$ at $37^{\circ} \mathrm{C}$, tubes were decanted and washed with PBS (pH 7.2) and dried. Tubes were then stained with crystal violet $0.1 \%$ for half an hour. Excess stain was removed and 
tubes were washed with deionized water and dried in an inverted position and observed for Biofilm formation. Biofilm formation was considered positive when visible film lined the wall and bottom of the tube. Tubes were examined and biofilm formation was graded as absent, moderate or strong. Experiments were performed in triplicate.

\section{Congo red agar method ${ }^{6}$}

Congo red agar (CRA) was prepared by supplementing brain heart infusion broth (BHI) with 5\% sucrose and Congo red. The medium was composed of BHI $(37 \mathrm{gms} / \mathrm{L})$, sucrose $(50 \mathrm{gms} / \mathrm{L})$, agar no.1 (10 gms/L) and congo red stain $(0.8 \mathrm{gms} / \mathrm{L})$. Congo red was prepared as concentrated aqueous solution and autoclaved at $121^{\circ} \mathrm{C}$ for 15 minutes and then added when the agar had cooled to $55^{\circ} \mathrm{C}$. The CRA plate was inoculated with the microorganism from an overnight broth culture and incubated aerobically at $37^{\circ} \mathrm{C}$ for 24 to 48 hours. Black colored colonies with dry crystalline consistency was interpreted as biofilm producing strains. Red colored colonies were interpreted as non-biofilm producing isolate. Experiments were performed in triplicate.

\section{Interpretation of biofilm formation}

\begin{tabular}{|l|l|l|}
\hline Mean OD & Adherence & Biofilm formation \\
\hline $\mathbf{< 0 . 1 2 0}$ & Non / weak & Non / weak \\
\hline $\begin{array}{l}\mathbf{0 . 1 2 0}- \\
\mathbf{0 . 0 2 4 0}\end{array}$ & Moderate & Moderate \\
\hline $\mathbf{> 0 . 2 4 0}$ & Strong & Strong \\
\hline
\end{tabular}

\section{Results and Discussion}

In the present study a total of 180 clinical isolates of $E$. coli were included for the detection of biofilm formation.
Out of the180 UPEC E. coli, 76 isolates were found to strongly positive for biofilm production, 54 were found to be moderately positive and 50 were weakly positive or negative for biofilm production by TCP method, 62 isolates were strongly positive, 45 moderately positive and 73 were weakly positive or non-biofilm producers by tube method similarly 68 isolates were strongly positive, 49 moderately positive and for biofilm and 63 were weakly positive or nonbiofilm producers by Congo red agar method (Table.1-3).

Only strongly positive and moderately positive and moderately positive isolates were considered as biofilm producers while weakly positive isolates were considered and nonbiofilm producers.

Tissue culture plate method was considered as the gold standard method for the detection of biofilm production.

Microbial biofilm is an assemblage of microorganisms which are irreversibly associated with a surface and enclosed in a polysaccharide matrix. Biofilm producing capacity acts as an important virulence factor in microorganisms as it provides a survival advantage to the organism producing it, thus it is difficult to treat biofilm associated infections than infections caused by planktonic bacteria.

Biofilm producing microorganisms are associated with nosocomial, implant associated infections, recurrent and persistent infections, biofilm forming isolates a thus it becomes necessary to evaluate the pathogens isolated for biofilm production to prevent treatment failure. 
Table.1 Biofilm production by Congo red agar method, tube method and tissue culture plate method

\begin{tabular}{|l|c|c|c|c|}
\hline Method & $\begin{array}{c}\text { Strongly } \\
\text { positive }\end{array}$ & $\begin{array}{c}\text { Moderately } \\
\text { positive }\end{array}$ & $\begin{array}{c}\text { Weakly } \\
\text { positive/negative }\end{array}$ & Total \\
\hline CRE & 68 & 49 & 63 & 180 \\
\hline TM & 62 & 45 & 73 & 180 \\
\hline TCP & $76(42.22)$ & $54(30)$ & $50(27.77)$ & 180 \\
\hline
\end{tabular}

Table.2 Biofilm producers by various phenotypic methods

\begin{tabular}{|l|c|c|c|}
\hline Method & $\begin{array}{c}\text { Biofilm producers, n } \\
(\boldsymbol{\%})\end{array}$ & $\begin{array}{c}\text { Biofilm non } \\
\text { producers, n }(\boldsymbol{\%})\end{array}$ & Total \\
\hline Congo red agar method & $117(65 \%)$ & $63(35 \%)$ & 180 \\
\hline Tube adherence method & $107(59.44 \%)$ & $73(23.88 \%)$ & 180 \\
\hline Tissue culture plate method & $130(72.22 \%)$ & $50(27.77 \%)$ & 180 \\
\hline
\end{tabular}

Table.3 Sensitivity, specificity, PPV and NPV of two phenotypic method of biofilm detection

\begin{tabular}{|l|c|c|c|c|}
\hline Method & Sensitivity & Specificity & PPV & NPV \\
\hline CRE & 81.52 & 78 & 90.59 & 61.90 \\
\hline TAM & 67.69 & 62 & 82.24 & 42.46 \\
\hline
\end{tabular}

In the present study, out of the 180 clinical isolates of E. coli, 130 isolates were found to be biofilm producers accounting to $72.22 \%$ of the isolates being biofilm producers by the TCP method which is considered as the gold standard in phenotypic method of biofilm detection $^{7,8,9}$, this is in correlation with a study conducted by Rashmi et al., ${ }^{10}$ and Sharma et al., ${ }^{11}$ who reports that $69 \%$ and $67.5 \%$ of the $E$. coli isolates from urine to be biofilm producers by TCP method.

Many surface determinants like type I fimbriae, autotransporter protein like $\mathrm{Ag} 43$, curlie fimbriae, $\mathrm{F}$ conjugative pilus and exopolysaccharide are associated with biofilm production. In the study $72.22 \%$ of the isolates were found to be biofilm producers by TCP method, $65 \%$ by Congo red agar method and $59.44 \%$ by tube adherence method. The ability of Congo red agar $(65 \%)$ for biofilm detection was similar to that of
TCP (72.22\%) method whereas tube adherence method detected only $59.44 \%$ of the isolates to be biofilm producers, these findings are in contrast to the findings by Taj Y et al., ${ }^{12}$ who reported only $3.47 \%$, Bos et al., ${ }^{13}$ who reported $6.15 \%$ and Deka et al., who reported $20 \%$ ability of CRE method to detect biofilm production by microorganisms. Saroj et al., ${ }^{14}$ and Munesh et al., ${ }^{15}$ reported high utility of CRE with $72 \%$ and $72.9 \%$ detection rate respectively. In the present study, tube adherence method showed only $59.44 \%$ of the isolates to be producing biofilm, this could be due to subjective difference in the interpretation, where as there was better correlation between TCP method and CRA method The basis of biofilm detection by Congo red agar method is that Congo red stain binds with the extracellular adhesive amyloid fibers termed curli that mediate adhesion and promote biofilm formation in $E$. $\operatorname{coli}^{16}$, so the biofilm detection 
rate is comparable with that of TCP method.

In conclusion, this study was aimed to include a simple and convenient method to screen microbial isolates for biofilm production. Considering the ease of doing the test, we conclude that both TCP method and CRA method of biofilm detection can be incorporated as a routine screening method for bio film detection.

\section{References}

1. Pinheiro L, Brito CI, Pereira VC, Oliveira A, Camargo CH, Cunha Mde L. Reduced susceptibility to vancomycin and biofilm formation in methicillin-resistant Staphylococcus epidermidis isolated from blood cultures. Mem Inst Oswaldo Cruz. 2014;109(7):871-878.

2. Stewart PS, Costerton JW. Antibiotic resistance of bacteria in biofilms. Lancet. 2001; 358(9276):135-138.

3. Costerton et al., 1999

4. Christensen GD, Simpson WA, Younger JJ, Baddour LM, Barrett FF, Melton DM, Beachey EH. Adherence of coagulasenegative staphylococci to plastic tissue culture plates: a quantitative model for the adherence of staphylococci to medical devices., J Clin Microbiol. 1985 Dec; 22(6):996-1006.

5. Christensen GD, Simpson WA, Bisno AL, et al., Adherence of slime-producing strains of Staphylococcus epidermidis to smooth surfaces. Infect Immun. 1982; 37:318-26.

6. Freeman DJ, Falkiner FR, Keane CT. New method for detecting slime production by coagulase negative staphylococci. J Clin Pathol. 1989; 42:872-4.

7. Tayal RA, Baveja SM, De AS. Analysis of biofilm formation and antibiotic susceptibility pattern of uropathogens in patients admitted in a tertiary care hospital in
India. Int J Health Allied Sci 2015; 4:247-52.

8. Hassan A, Usman J, Kaleem F, Omair M, Khalid A, Iqbal M. Evaluation of different detection methods of biofilm formation in the clinical isolates. Braz J Infect Dis 2011; 15(4):305-11.

9. Golia S, Hittinahalli V, Karjigi SK, Reddy KM. Correlation between biofilm formation of uropathogenic Escherichia coli and its antibiotic resistance pattern. Journal of evolution of Medical and dental Sciences 2012; 1(3):166-75.

10. Rashmi MK, Mahantesh BN. Characterization and antibiotic susceptibility pattern of coagulase negative staphylococci with special reference to methicillin resistance. Int J Curr Microbiol App Sci. 2016; 5:114-20.

11. Sharma M, Aparna, Yadav S, Chaudhary U. Biofilm production in uropathogenic Escherichia coli. Indian J Pathol Microbiol. 2009; 52:294.

12. Taj Y, Essa F, Aziz F, Kazmi SU. Study on biofilm forming properties of clinical isolates of Staphylococcus aureus. J Infect Dev Ctries 2012;5(6):403-09.

13.Bose S, Khodke M, Basak S, Mallick SK. Detection of biofilm producing Staphylococci: Need of the hour. J Clin Diagn Res 2009; 3:1915-20.

14. Saroj G, Vivek H, Sujata KK, Reddy M. Correlation between biofilm formation of uropathogenic Escherichia coli and its antibiotic resistance pattern. J Evol Med Dent Sci. 2012; 1:166-75.

15. Congo Red Interactions with Curli-Producing E. coli and Native Curli Amyloid FibersCourtney Reichhard, Amy N. Jacobson, Marie C. Maher, Jeremy Uang, Oscar A. McCrate, Michael Eckart, Lynette Cegelski. Congo Red Interactions with CurliProducing E. coli and Native Curli Amyloid Fibers. Plos one October 20, 2015. 


\section{How to cite this article:}

Rashmi P Mahale, M. N. Sumana, K. Anuradha and Adline Princy. 2020. Comparative Evaluation of Different Phenotypic Methods for Biofilm Formation among Uropathogenic $E$. coli (UPEC). Int.J.Curr.Microbiol.App.Sci. 9(07): 1094-1099. doi: https://doi.org/10.20546/ijcmas.2020.907.128 